\title{
REGIONAL ENLARGED CONTROLLABILITY FOR PARABOLIC SEMILINEAR SYSTEMS
}

\author{
Touria Karite $^{1}$, Ali Boutoulout ${ }^{2} \S$ \\ ${ }^{1,2}$ TSI Team, MACS Laboratory \\ Institute of Sciences \\ Moulay Ismail University \\ Meknes, MOROCCO
}

\begin{abstract}
The aim of this paper is to study the problem of the enlarged controllability for distributed parabolic semilinear systems evolving in spatial domain $\Omega$. It consists in finding the control $u$ with minimum cost that steers the system from the initial state $y_{0}$ to a state between two prescribed levels $l_{1}$ and $l_{2}$.

We give some definitions and properties concerning this concept and then we use two approaches to calculate the control $u$, the first one is based on the sub-differential method and the second one on the Lagrangian multiplier method. An algorithm is obtained and it's simulated numerically.
\end{abstract}

AMS Subject Classification: 93B05, 93C20

Key Words: distributed systems, parabolic systems, regional controllability, Lagrangian approach, sub-differential method, semilinear systems, Uzawa algorithm

\section{Introduction}

Parabolic problems are one of the fields of mathematics which undergoes a detailed investigation, due to the many problems which rely on this theory. Parabolic equations describe various time dependent models of many physical, chemical and biological phenomena. Such problems are reaction-diffusion and

Received: $\quad$ November 30, 2016

Revised: $\quad$ January 17, 2017

Published: $\quad$ February 28, 2017

$\S_{\text {Correspondence author }}$ (c) 2017 Academic Publications, Ltd. url: www.acadpubl.eu 
convection-diffusion systems, air pollution and meteorological models, flame propagation, superconductivity or Navier-Stokes and nonlinear heat equation, for more examples see [12].

Semilinear parabolic problems are a special kind of nonlinear equations. They arise in various physical and chemical problems, as well as their abstract form in applied mathematics. The parabolic semilinear problems can be treated as abstract ordinary differential equations, hence semigroup theory is used (see $[9,12,15])$.

A large part of physical systems are modeled by nonlinear equations for example, the equations governing a fluid submitted to thermo-diffusion and absorption effects in a porous medium; the species isolation in the fluid is governed at the local scale by a system of nonlinear parabolic equations. In this work, we are interested in the semilinear systems which have occupied an important place in control and systems theory. The study of these kind of systems has become fairly common, and is now an established area of research with a long list of publications. For example, Fabre et al. in [10] prove approximate controllability in $L^{p}(\Omega)$ for $1 \leq p \leq \infty$ using a control which can be internal or on the boundary and when the nonlinearity $\mathcal{N}(s)$ is globally Lipschitz. Bian [2] investigated the approximate controllability for a class of semilinear systems, Carmichael [5] used the Banach fixed-point theorem to obtain a local exact controllability in the case of nonlinearities with small Lipschitz constants, and Zhang [20] studied the local exact controllability of semi-linear evolution systems.

The reasons for studying this kind of problem are so many. An important advantage of this type of control is its ability to cope with hard constraints on controls and states. It has, therefore, been widely applied in petro-chemical and related industries where satisfaction of constraints is particularly important because efficiency demands operating points on or close to the boundary of the set of admissible states and controls. Also, the mathematical model of a real system is obtained from measures or from approximation techniques which are usually affected by perturbations, then if the solution for such system is approximately known, the control problem subject to output constraints is more realistic and more adapted for system analysis than the classical one.

The aim of this paper is to give an approach that leads to an algorithm for the computation of the optimal control which satisfies the output constraints. The paper is organized as follows:

In section two we present the problem statement for regional constrained controllability of semilinear systems, we give some definitions and properties. Section three is devoted to demonstrate some approaches which resolve the 
problem. In section four, we develop an algorithm that compute the control and we give some numerical results.

\section{Problem Statement}

Let $\Omega \in \mathbb{R}^{n}(n=1,2,3)$ denote a bounded set, with a regular boundary $\partial \Omega$. We use the notations $\left.Q_{T}=\Omega \times\right] 0, T$, where $0<T<+\infty$. Let $\Gamma_{T}=\partial \Omega \times[0, T]$, i.e. the lateral of $Q_{T}$. And we consider the following parabolic semilinear system:

$$
\begin{cases}\partial_{t} y(x, t)-\mathcal{A} y(x, t)-\mathcal{N} y(x, t)=B u(t) & Q_{T} \\ y(\xi, t)=0 & \Gamma_{T} \\ y(x, 0)=\varphi & \Omega\end{cases}
$$

where $\mathcal{A}$ is a second order linear differential operator which generates a strongly continuous semi-group $(S(t))_{t \geq 0}$ on Hilbert space $L^{2}(\Omega)$ and

$$
\mathcal{N}: L^{2}\left(0, T ; L^{2}(\Omega)\right) \longrightarrow L^{2}\left(0, T ; L^{2}(\Omega)\right)
$$

a locally Lipschitz continuous nonlinear operator (see [18]). $B \in \mathcal{L}\left(\mathbb{R}^{p}, L^{2}(\Omega)\right)$, $\varphi \in L^{2}(\Omega)$ and $u \in \mathcal{U}=L^{2}\left(0, T, \mathbb{R}^{p}\right)$ (where $p$ is the number of actuators).

Using the idea of Duhamel's principle, the solution of (1) could be written under the form:

$$
y_{u}(T)=S(T) \varphi+\int_{0}^{T} S(T-\tau) \mathcal{N} y(\tau) d \tau+\int_{0}^{T} S(T-\tau) B u(\tau) d \tau .
$$

With $y_{u} \in L^{2}\left(0, T ; L^{2}(\Omega)\right)($ see $[13])$.

Let $H$ be the operator from $\mathcal{U} \rightarrow L^{2}(\Omega)$ defined by:

$$
H u=\int_{0}^{T} S(T-s) B u(s) d s \quad \forall u \in \mathcal{U},
$$

We define the following operator:

$$
\begin{aligned}
G_{\omega}: L^{2}\left(0, T ; L^{2}(\Omega)\right) & \longrightarrow L^{2}\left(0, T ; L^{2}(\Omega)\right) \\
y(.) & \longmapsto \int_{0}^{T} S(T-\tau) \mathcal{N} y(\tau) d \tau .
\end{aligned}
$$

With all these notations, the solution of (1) could be written as follow:

$$
y_{u}(T)=S(T) \varphi+G_{\omega} y(.)+H u \text {. }
$$


For $\omega \subset \Omega$, open, nonempty and of positive Lebesgue measure, we consider the operator restriction:

$$
\begin{aligned}
\chi_{\omega}: L^{2}(\Omega) & \longrightarrow L^{2}(\omega) \\
y & \longmapsto \chi_{\omega} y=\left.y\right|_{\omega} .
\end{aligned}
$$

Its adjoint $\chi_{\omega}^{*}$ is given by:

$$
\left(\chi_{\omega}^{*} y\right)(x)=\left\{\begin{array}{lc}
y(x) & x \in \omega \\
0 & x \in \Omega \backslash \omega
\end{array}\right.
$$

Let $\alpha($.$) and \beta($.$) be two functions in L^{2}(\omega)$ such that $\alpha(.) \leq \beta($.$) a.e. in \omega$. Throughout the paper we set:

$$
[\alpha(.), \beta(.)]=\left\{y \in L^{2}(\omega) \quad \mid \quad \alpha(.) \leq y \leq \beta(.) \quad \text { a.e. in } \omega\right\} .
$$

Definition 1. We say that the system (1) is $[\alpha(),. \beta()$.$] -controllable in \omega$ if there exists $u \in \mathcal{U}$ such that $y_{u}(T)$ solution of (1) satisfies:

$$
\alpha(.) \leq y_{u}(T) \leq \beta(.) \quad \text { a.e. in } \omega .
$$

Then we have the following proposition:

\section{Proposition 2.}

The system (1) is $[\alpha(),. \beta()$.$] -controllable in \omega$ if and only if:

$$
\left([\alpha(.), \beta(.)] \backslash \chi_{\omega} S(T) y_{0}\right) \cap\left(\operatorname{Im} \chi_{\omega} G_{\omega}+\operatorname{Im} \chi_{\omega} H\right) \neq \emptyset .
$$

Proof. • Suppose that $\left([\alpha(),. \beta().] \backslash \chi_{\omega} S(T) y_{0}\right) \cap\left(\operatorname{Im} \chi_{\omega} G_{\omega}+\operatorname{Im} \chi_{\omega} H\right) \neq \emptyset$ then, there exists $z \in[\alpha(),. \beta().] \backslash \chi_{\omega} S(T) y_{0}$ such that $z \in \operatorname{Im} \chi_{\omega} G_{\omega}+\operatorname{Im} \chi_{\omega} H$ so $z=z_{1}+z_{2}$, such that $z_{1}=\chi_{\omega} G_{\omega} y$ with $y \in L^{2}\left(0, T ; L^{2}(\Omega)\right)$ and $\exists u \in \mathcal{U}$ such that $z_{2}=\chi_{\omega} H u$, then $z=\chi_{\omega}\left(G_{\omega} y+H u\right) \in[\alpha(),. \beta().] \backslash \chi_{\omega} S(T) y_{0}$, thus $\chi_{\omega} y_{u}(T) \in[\alpha(),. \beta()$.$] and finally (1)$ is $[\alpha(),. \beta()$.$] -controllable in \omega$.

- Suppose that the system (1) is exactly $[\alpha(),. \beta()$.$] -controllable in \omega$, which is equivalent to write $\chi_{\omega} y_{u}(T) \in[\alpha(),. \beta()$.$] .$

We have $\chi_{\omega} y_{u}(T)=\chi_{\omega} S(T) y_{0}+\chi_{\omega} G_{\omega} y()+.\chi_{\omega} H u$, let's denote by $z=$ $\chi_{\omega} y_{u}(T)-\chi_{\omega} S(T) y_{0}=z_{1}+z_{2}$.

It leads to $z_{1} \in \operatorname{Im}\left(\chi_{\omega} G_{\omega}\right)$ and $z_{2} \in \operatorname{Im}\left(\chi_{\omega} H\right)$.

Thus $z \in \operatorname{Im}\left(\chi_{\omega} G_{\omega}\right)+\operatorname{Im}\left(\chi_{\omega} H\right)$. And $z \in[\alpha(),. \beta().] \backslash \chi_{\omega} S(T) y_{0}$. Finally, we prove that

$$
\left([\alpha(.), \beta(.)] \backslash \chi_{\omega} S(T) y_{0}\right) \cap\left(\operatorname{Im} \chi_{\omega} G_{\omega}+\operatorname{Im} \chi_{\omega} H\right) \neq \emptyset .
$$


Remark 1. 1. If the system (1) is $[\alpha(),. \beta()$.$] -controllable in \omega_{1}$ then it's $[\alpha(),. \beta()$.$] -controllable in \omega_{2} \subset \omega_{1}$.

2. Let $J(u)=\frac{1}{2} \int_{0}^{T}\|u(t)\|_{\mathrm{R}^{p}}^{2} d t$ be the transfert cost, $z_{d} \in[\alpha(),. \beta()$.$] and$ consider the sets:

$$
W_{\omega}=\left\{u \in L^{2}\left(0, T ; \mathbb{R}^{p}\right) \quad \mid \quad y_{u}(T)=z_{d} \text { in } \omega\right\},
$$

and

$$
W_{[\alpha(.), \beta(.)]}=\left\{u \in L^{2}\left(0, T ; \mathbb{R}^{p}\right) \quad \mid \quad \alpha(.) \leq y_{u}(T) \leq \beta(.) \text { a.e. in } \omega\right\} .
$$

Then we have:

$$
\inf _{W_{[\alpha(\cdot), \beta(.)]}} J(u) \leq \inf _{W_{\omega}} J(u)
$$

This means that the cost of steering the system in $[\alpha(),. \beta()$.$] is less than$ steering it to a fixed desired state $z_{d} \in[\alpha(),. \beta()$.$] .$

Let's recall that an actuator is conventionally defined by a couple $(D, f)$, where $D$ is a nonempty closed part of $\bar{\Omega}$, and it represent the geometric support of the actuator. And $f \in L^{2}(D)$ define the spatial distribution of the action on the support $D$.

In the case of a pointwise actuator (internal or boundary) $D=\{b\}$ and $f=\delta(b-$.$) , where \delta$ is the Dirac mass concentrated in $b$, and the actuator is then denoted by $\left(b, \delta_{b}\right)$. For definitions and properties of strategic actuators we refer to $[19,8]$.

We also recall that the system (1) is said to be $\omega$-exactly (resp. $\omega$-weakly) controllable, if for all $y_{d} \in L^{2}(\omega)$ (resp. for all $\epsilon>0$ ) there exists a control $u \in \mathcal{U}$ such that $\chi_{\omega} y_{u}(T)=y_{d}\left(\operatorname{resp} .\left\|\chi_{\omega} y_{u}(T)-y_{d}\right\|_{L^{2}(\omega)} \leq \epsilon\right)($ see $[3])$.

Definition 3. The actuator $(D, f)$ is said to be $[\alpha(),. \beta()$.$] -strategic in \omega$ if the excited system is $[\alpha(),. \beta()$.$] -controllable in \omega$.

The $[\alpha(),. \beta()$.$] -controllability in \omega$ can be characterized by the following proposition:

Proposition 4. The system (1) is $[\alpha(),. \beta()$.$] -controllable in \omega$ if and only if:

$$
\left(\operatorname{Ker} \chi_{\omega}+\operatorname{Im} G_{\omega}+\operatorname{Im} H\right) \cap[\alpha(.), \beta(.)] \neq \emptyset .
$$

Proof. - We suppose that there exists $z \in[\alpha(),. \beta()$.$] , and u \in \mathcal{U}$ such that $\chi_{\omega} z_{u}(T)=\chi_{\omega} z$, let's consider $z_{1}=z-z_{u}(T)$ and $z_{2}=z_{u}(T)$, then: 
$z=z_{1}+z_{2}$ where $z_{1} \in \operatorname{Ker} \chi_{\omega}$ and $z_{2} \in \operatorname{Im} G_{\omega}+\operatorname{Im} H$. Which prove that $z \in\left(\operatorname{Ker} \chi_{\omega}+\operatorname{Im} G_{\omega}+\operatorname{Im} H\right)$.

- Conversely, we suppose that $\left(\operatorname{Ker} \chi_{\omega}+\operatorname{Im} G_{\omega}+\operatorname{Im} H\right) \cap[\alpha(),. \beta().] \neq \emptyset$ which means that there exists $z \in[\alpha(),. \beta()$.$] such that z \in \operatorname{Ker} \chi_{\omega}+\operatorname{Im} G_{\omega}+$ $\operatorname{Im} H$ so $z=z_{1}+z_{2}+z_{3}$, where $\chi_{\omega} z_{1}=0, \exists u \in \mathcal{U} \mid z_{2}=H u$ and $\exists y \in$ $L^{2}\left(0, T ; L^{2}(\Omega)\right) \mid z_{3}=G_{\omega} y$, then $\chi_{\omega} z=\chi_{\omega}\left(z_{1}+z_{2}+z_{3}\right)=\chi_{\omega} z_{2}+\chi_{\omega} z_{3}=$ $\chi_{\omega} H u+\chi_{\omega} G_{\omega} y$, which gives $\chi_{\omega} z \in \operatorname{Im} \chi_{\omega} H+\operatorname{Im} \chi_{\omega} G_{\omega}$. Finally, we have $\left(\operatorname{Im} \chi_{\omega} H+\operatorname{Im} \chi_{\omega} G_{\omega}\right) \cap[\alpha(),. \beta().] \neq \emptyset$. Thus (1) is $[\alpha(),. \beta()$.$] -controllable in$ $\omega$.

\section{Minimum Energy Control Subject to Output Constraints}

This section is devoted to explore two approaches that compute the optimal control which steers the system (1) from an initial state $y_{0}$ to a final one between $\alpha($.$) and \beta($.$) in a subregion \omega$. More accurately, we are interested in the following minimization problem:

$$
\left\{\begin{array}{l}
\inf \mathcal{J}(u) \\
u \in \mathcal{U}_{\text {ad }},
\end{array}\right.
$$

where $\mathcal{J}(u)=\frac{1}{2} \int_{0}^{T}\|u\|_{\mathrm{R}^{p}}^{2} d t$ and $\mathcal{U}_{a d}=\left\{u \in \mathcal{U} \mid \alpha(.) \leq y_{u}(T) \leq \beta(\right.$.$\left.) a.e. in \omega\right\}$ the set of admissible controls.

The following result ensure the existence and the uniqueness of the solution of the problem (7).

Proposition 5. If the system (1) is $[\alpha(),. \beta()$.$] -controllable in \omega$ then the problem (7) has a unique solution.

Proof. If the system (1) is $[\alpha(),. \beta()$.$] -controllable in \omega$, then $\mathcal{U}_{a d} \neq \emptyset$. Since the mapping $f: u \mapsto \frac{1}{2}\|u\|^{2}$ is strictly convex, coercive $\left(\lim _{\|u\| \rightarrow+\infty} f(u)=+\infty\right.$ for $u \in \mathcal{U}$ ), proper and lower semi-continuous in $\mathcal{U}$ which is reflexive, we have to verify that $\mathcal{U}_{a d}$ is closed convex subset of $\mathcal{U}$.

It is obvious that $\mathcal{U}_{a d}$ is convex. Indeed, for $(u, v) \in U^{2}$ :

$\alpha(.) \leq t y_{u}(T)+(1-t) y_{v}(T) \leq \beta($.$) .$

To prove that $\mathcal{U}_{a d}$ is closed, we consider a sequence $\left(u_{n}\right)_{n}$ in $\mathcal{U}_{\text {ad }}$ such that $u_{n} \rightarrow u$ strongly in $\mathcal{U}$. Since $\chi_{\omega} H$ and $\chi_{\omega} G_{\omega}$ are continuous then $\chi_{\omega} H u_{n} \rightarrow$ $\chi_{\omega} H u$ strongly in $L^{2}(\omega)$, but $\chi_{\omega} y_{u_{n}}(T) \in[\alpha(),. \beta()$.$] which is closed.$ 
Then $\chi_{\omega} y_{u_{n}}(T)=\chi_{\omega}\left(G_{\omega} y()+.H u_{n}\right) \in[\alpha(),. \beta()$.$] , then \chi_{\omega} y_{u}(T) \in[\alpha(),. \beta()$.$] ,$ which means that $u \in \mathcal{U}_{a d}$, hence $\mathcal{U}_{a d}$ is closed. And finally (7) has a unique solution.

Remark 2. The solution $u^{*}$ of (7) is characterized by $\left\langle u^{*}, u-u^{*}\right\rangle \geq$ $0 \forall u \in \mathcal{U}_{a d}$, but this characterization is hard to be implemented numerically. In the following, we will give two approaches characterizing the optimal control solution of (7).

\subsection{Subdifferential Approach}

The purpose of this subsection is to apply the subdifferential approach [1] to the optimal control problem for a parabolic equation excited by an internal zone actuator that steers the system (1) from an initial state $y_{0}$ to a final one between $\alpha($.$) and \beta($.$) .$

More precisely, we are interested to solve the problem of minimization (7).

Let us denote by:

- $\Gamma_{0}(U)$ the set of functions $\left.\left.f: U \mapsto \widetilde{\mathbb{R}}=\right]-\infty,+\infty\right]$ which are proper, lower semi-continuous (l.s.c) and convex in $\mathrm{U}$.

- For $f \in \Gamma_{0}(U)$

$$
\operatorname{dom}(f)=\{u \in U \mid f(u)<\infty\},
$$

is the domain of $f$ and $f^{*}$ its polar function given by:

$$
f^{*}\left(v^{*}\right)=\sup _{u \in \operatorname{dom}(f)}\left\{\left\langle v^{*}, u\right\rangle-f(u)\right\} \quad \forall v^{*} \in U .
$$

- For $v_{0} \in \operatorname{dom}(f)$, the sub-differential of $f$ at $v_{0}$ is given by the set:

$$
\partial f\left(v_{0}\right)=\left\{u^{*} \in U \quad \mid \quad f(u) \geq f\left(v_{0}\right)+\left\langle u^{*}, u-v_{0}\right\rangle, \quad \forall u \in U\right\} .
$$

- For every $u \in U$, we denote $\sigma(u)=\frac{1}{2}\|u\|^{2}$.

- For $K$ a nonempty subset of $U$

$$
\Psi_{K}(u)= \begin{cases}0 & \text { if } u \in K \\ +\infty & \text { if not, }\end{cases}
$$

denotes the indicator functional of $K$.

With all these notations, the problem (7) is equivalent to the following problem:

$$
\left\{\begin{array}{l}
\inf \left(\sigma(u)+\Psi_{\mathcal{U}_{a d}}(u)\right) \\
u \in \mathcal{U}
\end{array}\right.
$$


And the solution of the problem (8) could be characterized by the following result:

Proposition 6. If the system (1) is $[\alpha(),. \beta()$.$] -controllable in \omega$ then $u^{*}$ is the solution of (8) if and only if:

$$
u^{*} \in \mathcal{U}_{a d} \quad \text { and } \quad \Psi_{\mathcal{U}_{a d}}^{*}\left(-u^{*}\right)=-\left\|u^{*}\right\|^{2} .
$$

Proof. We suppose that $u^{*}$ is a solution of (8) which is equivalent to $0 \in$ $\partial\left(\sigma+\Psi_{u_{a d}}\right)\left(u^{*}\right)$, with $\sigma\left(u^{*}\right)=\frac{1}{2}\left\|u^{*}\right\|^{2}$.

We have $\sigma \in \Gamma_{0}(U)$ and since $\mathcal{U}_{a d}$ is closed, convex and nonempty, we obtain $\Psi_{\mathcal{u}_{a d}} \in \Gamma_{0}(U)$.

Furthermore, according to the hypothesis of the $[\alpha(),. \beta()$.$] -controllability$ in $\omega$, we have $\operatorname{dom} \sigma \cap \operatorname{dom} \Psi_{\mathcal{u}_{a d}} \neq \emptyset$. Since $\sigma$ is continuous we obtain

$$
\partial\left(\sigma+\Psi_{\mathcal{u}_{a d}}\right)\left(u^{*}\right)=\partial \sigma\left(u^{*}\right)+\partial \Psi_{\mathcal{u}_{a d}}\left(u^{*}\right) .
$$

It follows that $u^{*}$ is the solution of (8) if and only if $0 \in\left(\partial \sigma\left(u^{*}\right)+\partial \Psi_{u_{a d}}\left(u^{*}\right)\right)$.

Moreover, $\sigma$ is Fréchet-differentiable, so $\partial \sigma\left(u^{*}\right)=\left\{\nabla \sigma\left(u^{*}\right)\right\}=\left\{u^{*}\right\}$ and $u^{*}$ is the solution of $(8)$ if and only if $-u^{*} \in \partial \Psi_{\mathcal{u}_{a d}}\left(u^{*}\right)$ which is equivalent to $u^{*} \in \mathcal{U}_{a d}$ and $\Psi_{\mathcal{U}_{a d}}\left(u^{*}\right)+\Psi_{\mathcal{U}_{a d}}^{*}\left(-u^{*}\right)=-\left\|u^{*}\right\|^{2}$ which finally gives $u^{*} \in \mathcal{U}_{a d}$ and $\Psi_{\mathcal{u}_{a d}}^{*}\left(-u^{*}\right)=-\left\|u^{*}\right\|^{2}$.

We set

$$
\begin{aligned}
& a(.)=\alpha(.)-\chi_{\omega} S(T) y_{0}-\chi_{\omega} G_{\omega} y(.) \\
& b(.)=\beta(.)-\chi_{\omega} S(T) y_{0}-\chi_{\omega} G_{\omega} y(.) .
\end{aligned}
$$

Then $\mathcal{U}_{a d}=\left\{u \in \mathcal{U} \mid \chi_{\omega} H u \in[\alpha(),. \beta()].\right\}$ and we have the following result:

Proposition 7. If the system (1) is $[\alpha(),. \beta()$.$] -controllable in \omega$, then $u^{*}$ is the solution of (8) if and only if:

$$
\min \left\{\left\langle\left(\chi_{\omega} H\right)^{\dagger} a(.), u^{*}\right\rangle,\left\langle\left(\chi_{\omega} H\right)^{\dagger} b(.), u^{*}\right\rangle\right\}=\left\|u^{*}\right\|^{2},
$$

where $\left(\chi_{\omega} H\right)^{\dagger}$ is the pseudo-inverse of $\chi_{\omega} H$.

Proof. We have $\mathcal{U}_{a d}=\left(\chi_{\omega} H\right)^{\dagger}[a(),. b()$.$] and from the proposition (6), u^{*}$ is a solution of (8) if and only if $u^{*} \in \mathcal{U}_{a d}$ and $\Psi_{\mathcal{u}_{a d}}^{*}\left(-u^{*}\right)=-\left\|u^{*}\right\|^{2}$. 
Then

$$
\begin{aligned}
\Psi_{\mathcal{U}_{a d}}^{*}\left(v^{*}\right) & =\sup _{v \in \mathcal{U}_{a d}}\left\langle v^{*}, v\right\rangle \quad \forall v^{*} \in \mathcal{U} \\
& =\sup _{v \in\left(\chi_{\omega} H\right)^{\dagger}([a(.), b(.)])}\left\langle v^{*}, v\right\rangle \\
& =\sup _{y \in[a(.), b(.)]}\left\langle v^{*},\left(\chi_{\omega} H\right)^{\dagger} y\right\rangle \\
& =\sup _{\lambda \in[0,1]}\left\langle\left(\left(\chi_{\omega} H\right)^{\dagger}\right)^{*} v^{*}, \lambda a(.)+(1-\lambda) b(.)\right\rangle
\end{aligned}
$$

Since the map $\lambda \mapsto\left\langle\left(\left(\chi_{\omega} H\right)^{\dagger}\right)^{*} v^{*}, \lambda a()+.(1-\lambda) b().\right\rangle$ from $[0,1] \subset L^{2}(\omega)$ into $\mathbb{R}$ is convex and continuous, we obtain according to the Krein-Milman theorem $[17]$ :

$$
\Psi_{\mathcal{U}_{a d}}^{*}\left(v^{*}\right)=\sup _{\lambda \in[0,1]}\left\langle\left(\left(\chi_{\omega} H\right)^{\dagger}\right)^{*} v^{*}, \lambda a(.)+(1-\lambda) b(.)\right\rangle .
$$

It follows that $\Psi_{\mathcal{U}_{a d}}^{*}\left(v^{*}\right)=\max \left\{\left\langle v^{*},\left(\chi_{\omega} H\right)^{\dagger} a().\right\rangle,\left\langle v^{*},\left(\chi_{\omega} H\right)^{\dagger} b().\right\rangle\right\}$ and from proposition (6) we obtain (10).

Remark 3. If $\alpha()=.\beta()=.\left\{y_{d}\right\}$ we find the notion of regional controllability then

$$
a(.)=b(.)=y_{d}-\chi_{\omega} S(T) y_{0}-\chi_{\omega} G_{\omega} y(.)
$$

and

$$
\mathcal{U}_{a d}=\left(\chi_{\omega} H\right)^{\dagger}\left(y_{d}-\chi_{\omega} S(T) y_{0}-\chi_{\omega} G_{\omega} y(.)\right)
$$

and the solution of (8) is given by:

$$
u^{*}(t)=\left(\chi_{\omega} H\right)^{\dagger}\left(y_{d}-\chi_{\omega} S(T) y_{0}-\chi_{\omega} G_{\omega} y(.)\right) \text {. }
$$

This approach is hard to implement numerically. That's why we are exposing in the next paragraph an approach which can be implemented in numerical examples.

\subsection{Lagrangian Multiplier Approach}

The purpose of this paragraph is to explore an approach devoted to solve optimisation problems with constraints. It compute the optimal control which steers the system in (1) from $y_{0}$ to a final state between $\alpha($.$) and \beta($.$) in a$ subregion $\omega$.

Consider the problem (7) when the system (1) is excited by a zone actuator $(D, f)$. The following result gives a useful characterization of the problem $(7)$ : 
Theorem 8. If the actuator $(D, f)$ is $[\alpha(),. \beta()$.$] -strategic in \omega$ then the solution of (7) is given by:

$$
u^{*}=-\left(\chi_{\omega} H\right)^{*} \lambda^{*}
$$

where $\lambda^{*}$ is the solution of:

$$
\left\{\begin{array}{l}
z_{d}^{*}=P_{[\alpha(.), \beta(.)]}\left(\rho \lambda^{*}+z_{d}^{*}\right) \\
z_{d}^{*}+R_{\omega} \lambda^{*}=\chi_{\omega}\left[S(T) y_{0}+G_{\omega} y(.)\right]
\end{array}\right.
$$

while $P_{[\alpha(.), \beta(.)]}: L^{2}(\omega) \rightarrow[\alpha(),. \beta()$.$] denotes the projection operator, \rho>0$ and $R_{\omega}=\left(\chi_{\omega} H\right)\left(\chi_{\omega} H\right)^{*}$.

Proof. If the actuator $(D, f)$ is $[\alpha(),. \beta()$.$] -strategic in \omega$ then $\mathcal{U}_{a d} \neq \emptyset$ and (7) has a unique solution. The problem (7) is equivalent to the following saddle point problem:

$$
\left\{\begin{array}{l}
\inf \frac{1}{2}\|u\|^{2} \\
\left(u, z_{d}\right) \in W
\end{array}\right.
$$

where $W=\left\{\left(u, z_{d}\right) \in \mathcal{U} \times[\alpha(),. \beta()] \mid. \chi_{\omega} y_{u}(T)=z_{d}\right\}$.

We associate to the problem (13) the Lagrangian functional L defined by:

For $\left(u, z_{d}, \lambda\right) \in \mathcal{U} \times[\alpha(),. \beta().] \times L^{2}(\omega)$

$$
L\left(u, z_{d}, \lambda\right)=\frac{1}{2}\|u\|^{2}+\left\langle\lambda, \chi_{\omega} y_{u}(T)-z_{d}\right\rangle_{L^{2}(\omega)},
$$

where $\langle., .\rangle_{L^{2}(\omega)}$ is the scalar product in $L^{2}(\omega)$.

Let us recall that $\left(u^{*}, z_{d}^{*}, \lambda^{*}\right)$ is a saddle point of $\mathrm{L}$ if:

$$
\max _{\lambda \in L^{2}(\omega)} L\left(u^{*}, z_{d}^{*}, \lambda\right)=L\left(u^{*}, z_{d}^{*}, \lambda^{*}\right)=\min _{u \in \mathcal{U}} L\left(u, z_{d}, \lambda^{*}\right) .
$$

For more details about the saddle point we refer to [14].

- Let's prove that L admits a saddle point.

The set $\mathcal{U} \times[\alpha(),. \beta()$.$] is nonempty, closed and convex. Obviously, the map$ $\left(u, z_{d}\right) \mapsto L\left(u, z_{d}, \lambda\right)$ is convex and lower semi-continuous for every $\lambda \in L^{2}(\omega)$. In addition, for every $\left(u, z_{d}\right) \in \mathcal{U} \times[\alpha(),. \beta()$.$] , the map \lambda \mapsto L\left(u, z_{d}, \lambda\right)$ is concave 
and upper semi-continuous. On the other hand, there exists $\lambda_{0} \in L^{2}(\omega)$ such that

$$
\lim _{\left\|\left(u, z_{d}\right)\right\| \rightarrow+\infty} L\left(u, z_{d}, \lambda_{0}\right)=+\infty,
$$

and there exists $\left(u_{0}, z_{d}^{0}\right) \in U \times[\alpha(),. \beta()$.$] such that:$

$$
\lim _{\|\lambda\| \rightarrow+\infty} L\left(u_{0}, z_{d}^{0}, \lambda\right)=-\infty
$$

This show that $\mathrm{L}$ admits a saddle point.

- Let's prove that $u^{*}$ is the solution of $(7)$.

We suppose that $\left(u^{*}, z_{d}^{*}, \lambda^{*}\right)$ is a saddle point of $\mathrm{L}$, we have:

$$
\begin{aligned}
& L\left(u^{*}, z_{d}^{*}, \lambda\right) \leq L\left(u^{*}, z_{d}^{*}, \lambda^{*}\right) \\
& \quad \leq L\left(u, z_{d}, \lambda^{*}\right) \quad \forall\left(u, z_{d}, \lambda\right) \in \mathcal{U} \times[\alpha(.), \beta(.)] \times L^{2}(\omega) .
\end{aligned}
$$

From the first inequality of (16), we have:

$$
\begin{aligned}
\frac{1}{2}\left\|u^{*}\right\|^{2}+\left\langle\lambda, \chi_{\omega} y_{u^{*}}(T)-z_{d}^{*}\right\rangle_{L^{2}(\omega)} \leq \frac{1}{2}\left\|u^{*}\right\|^{2}+\left\langle\lambda^{*}, \chi_{\omega} y_{u^{*}}(T)-z_{d}^{*}\right\rangle_{L^{2}(\omega)} \\
\forall \lambda \in L^{2}(\omega),
\end{aligned}
$$

which leads to: $\left\langle\lambda, \chi_{\omega} y_{u^{*}}(T)-z_{d}^{*}\right\rangle_{L^{2}(\omega)} \leq\left\langle\lambda^{*}, \chi_{\omega} y_{u^{*}}(T)-z_{d}^{*}\right\rangle_{L^{2}(\omega)} \forall \lambda \in L^{2}(\omega)$, it implies that $\chi_{\omega} y_{u^{*}}(T)=z_{d}^{*}$, hence $\chi_{\omega} y_{u^{*}}(T) \in[\alpha(),. \beta()$.$] .$

From the second inequality of (16), it follows that:

$$
\begin{aligned}
\frac{1}{2}\left\|u^{*}\right\|^{2}+\left\langle\lambda^{*}\right. & \left., \chi_{\omega} y_{u^{*}}(T)-z_{d}^{*}\right\rangle_{L^{2}(\omega)} \\
& \leq \frac{1}{2}\|u\|^{2}+\left\langle\lambda^{*}, \chi_{\omega} y_{u}(T)-z_{d}\right\rangle_{L^{2}(\omega)} \forall\left(u, z_{d}\right) \in \mathcal{U} \times[\alpha(.), \beta(.)] .
\end{aligned}
$$

Although, $\chi_{\omega} y_{u^{*}}(T)=z_{d}^{*}$, which leads to the following result:

$$
\frac{1}{2}\left\|u^{*}\right\|^{2} \leq \frac{1}{2}\|u\|^{2}+\left\langle\lambda^{*}, \chi_{\omega} y_{u}(T)-z_{d}\right\rangle_{L^{2}(\omega)} \forall\left(u, z_{d}\right) \in \mathcal{U} \times[\alpha(.), \beta(.)] .
$$

Taking $z_{d}=\chi_{\omega} y_{u}(T) \in[\alpha(),. \beta()$.$] , we obtain:$

$$
\frac{1}{2}\left\|u^{*}\right\|^{2} \leq \frac{1}{2}\|u\|^{2}
$$

which implies that $u^{*}$ is of minimum energy. 
- $\left(u^{*}, z_{d}^{*}, \lambda^{*}\right)$ is a saddle point of $\mathrm{L}$, then the following assumptions hold:

$$
\begin{gathered}
\left\langle u^{*}, u-u^{*}\right\rangle+\left\langle\lambda^{*}, \chi_{\omega} H\left(u-u^{*}\right)\right\rangle=0 \quad \forall u \in \mathcal{U}, \\
\left\langle\lambda^{*}, z_{d}-z_{d}^{*}\right\rangle \leq 0 \quad \forall z_{d} \in[\alpha(.), \beta(.)], \\
\left\langle\lambda-\lambda^{*}, \chi_{\omega} y_{u^{*}}(T)-z_{d}^{*}\right\rangle=0 \quad \forall \lambda \in L^{2}(\omega) .
\end{gathered}
$$

More details on the saddle point theory and its applications can be found in $[4,11,16]$.

From (17) we have $\left\langle u^{*}, u-u^{*}\right\rangle+\left\langle\lambda^{*}, \chi_{\omega} H\left(u-u^{*}\right)\right\rangle=0 \quad \forall u \in U$ which leads to:

$$
\left\langle u^{*}, u-u^{*}\right\rangle+\left\langle\left(\chi_{\omega} H\right)^{*} \lambda^{*}, u-u^{*}\right\rangle=0 \quad \forall u \in \mathcal{U} .
$$

Then we deduce that: $u^{*}+\left(\chi_{\omega} H\right)^{*} \lambda^{*}=0$, and finally we prove (11). So we have

$$
u^{*}=-\left(\chi_{\omega} H\right)^{*} \lambda^{*} .
$$

The equation (19) is equivalent to $\chi_{\omega} y_{u^{*}}(T)-z_{d}^{*}=0$. And since $y_{u^{*}}=S(T) y_{0}+$ $G_{\omega} y()+.H u^{*}$, we have:

$$
\chi_{\omega}\left[S(T) y_{0}+G_{\omega} y(.)+H u^{*}\right]-z_{d}^{*}=0 .
$$

Then $\chi_{\omega}\left[S(T) y_{0}+G_{\omega} y().\right]+\chi_{\omega} H\left(u^{*}\right)=z^{*}$, and with (11) we have:

$$
\chi_{\omega}\left[S(T) y_{0}+G_{\omega} y(.)\right]+\chi_{\omega} H\left(-\left(\chi_{\omega} H\right)^{*} \lambda^{*}\right)=z_{d}^{*} .
$$

Finally we obtain the second part of the system (12):

$$
\chi_{\omega}\left[S(T) y_{0}+G_{\omega} y(.)\right]-\left(\chi_{\omega} H\right)\left(\chi_{\omega} H\right)^{*} \lambda^{*}=z_{d}^{*},
$$

and it could be written as follow:

$$
\chi_{\omega}\left[S(T) y_{0}+G_{\omega} y(.)\right]=R_{\omega} \lambda^{*}+z_{d}^{*} \quad \text { where } \quad R_{\omega}=\left(\chi_{\omega} H\right)\left(\chi_{\omega} H\right)^{*} .
$$

From the inequality (18) we obtain $\left\langle\lambda^{*}, z_{d}-z_{d}^{*}\right\rangle \leq 0 \quad \forall z_{d} \in[\alpha(),. \beta()$.$] . Which$ leads to the following result:

$$
\left\langle\left(\rho \lambda^{*}+z_{d}^{*}\right)-z_{d}^{*}, z_{d}-z_{d}^{*}\right\rangle \leq 0 \quad \forall z_{d} \in[\alpha(.), \beta(.)] \forall \rho>0 .
$$

And finally we obtain: $z_{d}^{*}=P_{[\alpha(\cdot), \beta(.)]}\left(\rho \lambda^{*}+z_{d}^{*}\right)$. So we prove the first part of (12).

Corollary 9. If the system (1) is exactly controllable in $\omega$ and $\rho$ suitably chosen, then the system (12) has only one solution $\left(\lambda^{*}, z_{d}^{*}\right)$. 
Proof. The regional exact controllability in $\omega$ implies that $\left(\chi_{\omega} H\right)^{*}$ and $R_{\omega}$ are bijective. So if $\left(u^{*}, z_{d}^{*}, \lambda^{*}\right)$ is a saddle point of $\mathrm{L}$ then the system (12) is equivalent to

$$
\left\{\begin{array}{l}
\lambda^{*}=R_{\omega}^{-1}\left[\chi_{\omega}\left(S(T) y_{0}+G_{\omega} y(.)\right)-z_{d}^{*}\right] \\
z_{d}^{*}=P_{[\alpha(.), \beta(.)]}\left(-\rho R_{\omega}^{-1} z_{d}^{*}+\rho R_{\omega}^{-1} \chi_{\omega}\left(S(T) y_{0}+G_{\omega} y(.)\right)+z_{d}^{*}\right) .
\end{array}\right.
$$

It follows that $z_{d}^{*}$ is a fixed point of the function

$$
\begin{aligned}
F_{\rho}:[\alpha(.), \beta(.)] & \longrightarrow[\alpha(.), \beta(.)] \\
y & \longmapsto P_{[\alpha(.), \beta(.)]}\left(-\rho R_{\omega}^{-1} y+\rho R_{\omega}^{-1} \chi_{\omega}\left(S(T) y_{0}+G_{\omega} y(.)\right)+y\right) .
\end{aligned}
$$

The operator $R_{\omega}^{-1}$ is coercive, i.e.

$$
\exists m>0 \quad \text { such that }\left\langle R_{\omega}^{-1} y, y\right\rangle \geq m\|y\|^{2} \quad \forall y \in L^{2}(\omega) .
$$

It follows that

$$
\begin{aligned}
\left\|F_{\rho}(y)-F_{\rho}(z)\right\|^{2} & \leq\left\|-\rho R_{\omega}^{-1}(y-z)+(y-z)\right\|^{2} \\
& \leq \rho^{2}\left\|R_{\omega}^{-1}\right\|^{2}\|y-z\|^{2}+\|y-z\|^{2}-2 \rho\left\langle R_{\omega}^{-1}(y-z),(y-z)\right\rangle \\
& \leq \rho^{2}\left\|R_{\omega}^{-1}\right\|^{2}\|y-z\|^{2}+\|y-z\|^{2}-2 \rho m\|y-z\|^{2} \\
& \leq\left(1+\rho^{2}\left\|R_{\omega}^{-1}\right\|^{2}-2 \rho m\right)\|y-z\|^{2} \quad \forall y, z \in[\alpha(.), \beta(.)]
\end{aligned}
$$

and we deduce that if $0<\rho<\frac{2 m}{\left\|R_{\omega}^{-1}\right\|^{2}}$, then $F_{\rho}$ is a contraction mapping. This implies the uniqueness of $z^{*}$ et $\lambda^{*}$.

Remark 4. If $\alpha()=.\beta()=.y_{d}$ then $\mathcal{U}_{a d}=\left\{u \in \mathcal{U} \mid y_{u}(T)=\right.$ $y_{d}$ a.e. in $\left.\omega\right\}$, we find the notion of regional controllability and the solution of (7) is given by

$$
u^{*}(t)=\left(\chi_{\omega} H\right)^{*} R_{\omega}^{-1}\left[\alpha(.)-\chi_{\omega}\left(S(T) y_{0}+G_{\omega} y(.)\right)\right] .
$$

\section{Numerical Approach}

From the theorem (8) it follows that the solution of the problem (7) arises to compute the saddle points of $\mathrm{L}$ which is equivalent to solving the problem

$$
\inf _{\left(u, z_{d}\right) \in \mathcal{U} \times[\alpha(.), \beta(.)]}\left(\sup _{\lambda \in L^{2}(\omega)} L\left(u, z_{d}, \lambda\right)\right)
$$


To achieve this we shall use the following algorithm based on the algorithm of Uzawa type (see [11]):

\section{Algorithm}

Step 1: initialization.

$\Theta \Theta$ Choose two functions $\left(z_{d}^{0}, \lambda_{1}\right) \in[\alpha(),. \beta().] \times L^{2}(\omega)$,

$\ominus$ choose a region $\omega$, time $T$, and the position $b$ of the actuator, $\ominus$ choose the threshold accuracy $\epsilon$ small enough.

Step 2: Until $\left\|\lambda_{n+1}-\lambda_{n}\right\| \leq \epsilon$, repeat

$$
\begin{array}{l|l}
\ominus \text { Solve equation } u_{n}=-\left(\chi_{\omega} H\right)^{*} \lambda_{n}, \\
\ominus \text { Solve equation } z_{d}^{n}=P_{[\alpha(\cdot), \beta(.)]}\left(\rho \lambda_{n}+z_{d}^{n-1}\right), \\
\odot \text { Calculate } \lambda_{n+1} \text { by the formula } \lambda_{n+1}=\lambda_{n}+\left(\chi_{\omega} y_{u_{n}}(T)-z_{d}^{n}\right) .
\end{array}
$$

Step 3: Let $\left(u^{*}, z_{d}^{*}, \lambda^{*}\right)$ be a saddle point of $L$, then the sequence $u_{n}$ converges to $u^{*}$ solution of the problem (7) and the sequence $z_{n}$ converges to $z^{*}$.

Example 1. Let's consider $\Omega=] 0,1[$, and the following system:

$$
\begin{cases}\frac{\partial y(x, t)}{\partial t}=\mathcal{A} y(x, t)+c y^{2}(x, t)+B u(t) & ] 0,1[\times] 0, T[ \\ y(0, t)=y(1, t) & ] 0, T[ \\ y(x, 0)=0 & ] 0,1[\end{cases}
$$

where $\mathcal{A}=\Delta=\frac{\partial^{2}}{\partial x^{2}}, c=0.01$ and $B u(t)=\delta(x-b) u(t)$.

Let $b=0.5$ be the control action point and $\mathrm{T}=2$. Let's consider $\alpha(x)=$ $x^{2}(x-1)^{2}, \beta(x)=-2 x(x-1)$ and $\epsilon=10^{-4}$. Let $\left(\varphi_{i}\right)_{i}$ be the eigenfunctions of $\mathcal{A}$ associated with the eigenvalues $\gamma_{i}$, where

$$
\varphi_{i}=\sqrt{2} \sin (i \pi x) \text { and } \gamma_{i}=-(i \pi)^{2}
$$

Applying the previous algorithm we obtain the following results:

For $\underline{\omega=\Omega \text { : }}$ 

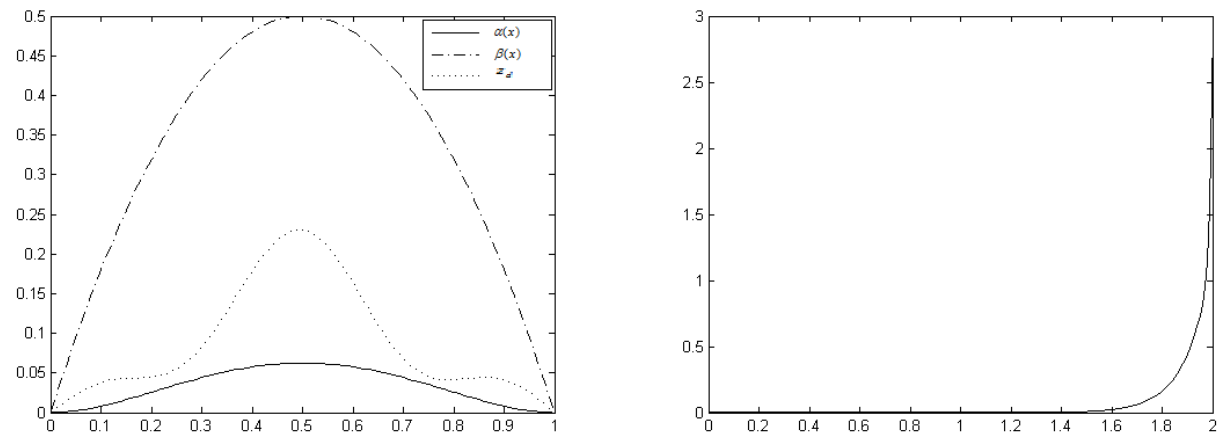

Figure 1: Reached state between $\alpha($.$) and \beta($.$) \quad Figure 2: Control function$

Figure 1 shows that the reached state is between $[\alpha(),. \beta()$.$] in the whole do-$ main so the actuator is $[\alpha(),. \beta()$.$] -strategic in \Omega$, and it's obtained with transfer cost $\left\|u^{*}\right\|^{2}=0.12$. Figure 2 shows the control function.

For $\omega=] 0.4,0.6[$ :
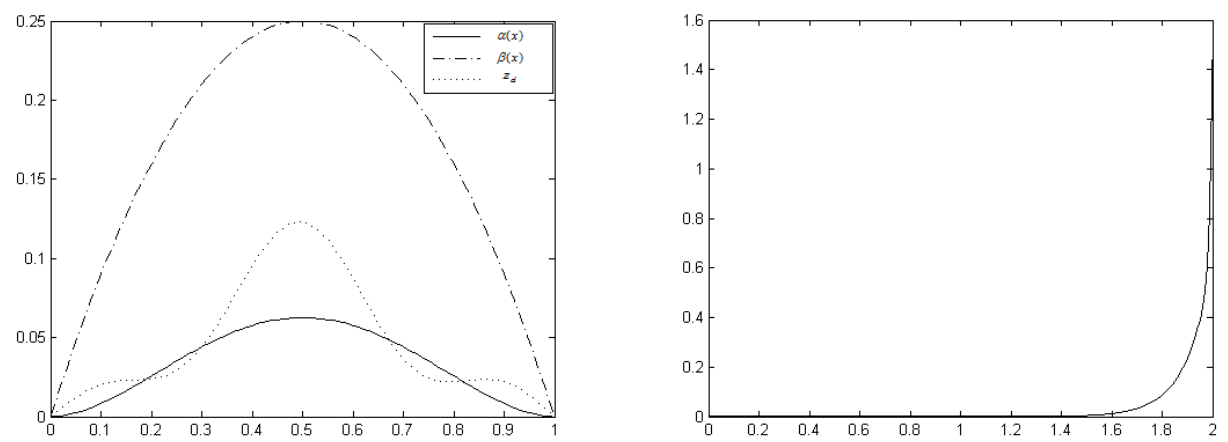

Figure 3: Reached state between $\alpha($.$) and \beta($.$) \quad Figure 4: Control function$

Figure 3 shows that the reached state is between $\alpha($.$) and \beta($.$) in the sub-$ region $\omega=] 0.4,0.6\left[\right.$ and is obtained with transfer cost $\left\|u^{*}\right\|^{2}=3.57 \times 10^{-2}$ with an error of order $\epsilon=8.37 \times 10^{-5}$. Figure 4 shows the control function. 
For $\omega=] 0.4,0.7[$ :
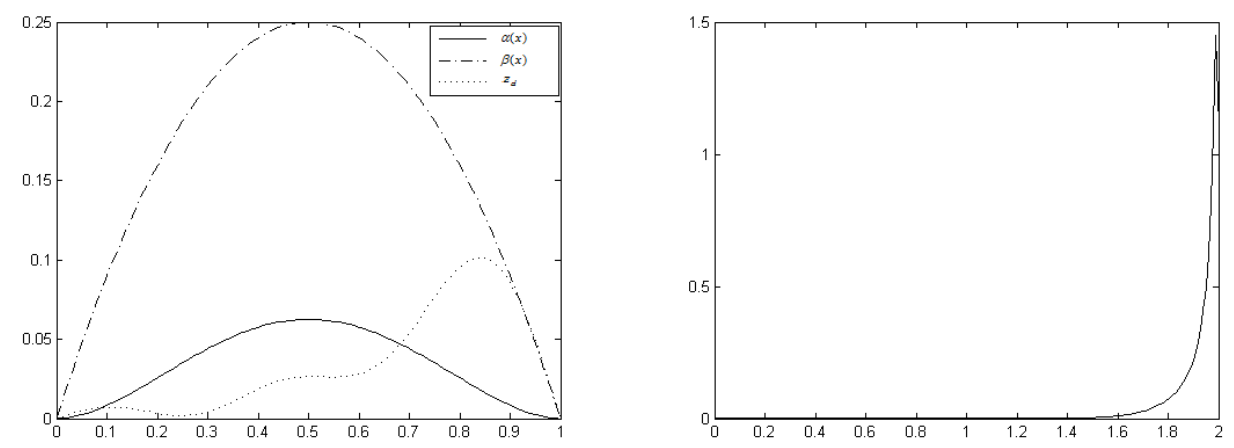

Figure 5: Reached state and $\alpha(),. \beta($.

Figure 6: Control function

If the actuator is located in $\mathrm{b}=0.87$, figure 5 is showing that the final state is not between $\alpha(x)$ and $\beta(x)$ in ]0.4, 0.7[. This means that the actuator located in $\mathrm{b}=0.87$ is not $[\alpha(),. \beta()$.$] -strategic in \omega$. Figure 6 is illustrating the control function.

\section{Conclusion}

We have developed an extension of the notion of controllability for parabolic semi-linear systems with constraints, the optimal control has been characterized using the Lagrangian approach. Interesting results are obtained and illustrated with numerical example and simulations. Future works aim to extend this notion of regional controllability with constrained in $\Gamma$ a part of the boundary $\partial \Omega$ of the evolution domain $\Omega$.

\section{Acknowledgments}

This work has been carried out with a grant from Hassan II Academy of Sciences and Technology.

\section{References}

[1] J.P. Aubin, L'analyse Non Linéaire et ses Motivations Économiques, Dunod, 1984.

[2] W.M. Bian, Constrained controllability of some Non-linear systems, Applicable Analysis, 72, No. 1-2 (1999), 57-73, doi: 10.1080/00036819908840730. 
[3] A. Boutoulout, A. Kamal and S.A.O. Beinane, Regional Controllability of Semi-Linear Distributed Parabolic Systems: Theory and Simulation, Intelligent Control and Automation, Vol, No. 3 (2012), 146-158, doi: 10.4236/ica.2012.32017.

[4] F. Brezzi and M. Fortin, Mixed and Hybrid Finite Element Methods, Springer-Verlag, New York (1991), doi: 10.1007/978-1-4612-3172-1.

[5] N. Carmichael and M.D. Quinn, Fixed-Point Methods in Nonlinear Control, Lecture notes in Control and Information Sciences, Springer-Verlag, Berlin Heidelberg (1984), doi: 10.1007/BFb0005643.

[6] R.F. Curtain and H. Zwart, An introduction to infinite dimensional linear systems theory, Springer-Verlag, New York (1995), doi: 10.1007/978-1-4612-4224-6.

[7] A. El Jai, A.J. Pritchard, M.C. Simon and E. Zerrik, Regional controllability of distributed parameter systems, International Journal of Control, 62, (1995), 1351-1365, doi: $10.1080 / 00207179508921603$.

[8] A. El Jai and A.J. Pritchard, Sensors and actuators in distributed systems analysis, Wiley, New York (1988).

[9] K.J. Engel, R. Nagel, One-Parameter Semigroups for Linear Evolution Equations, Springer-Verlag, New York (1999), doi: 10.1007/b97696.

[10] C. Fabre, P.J. Puel and E. Zuazua, Approximate controllability of the semilinear heat equation, Proc. Roy Soc. Edinburgh 125 A, (1995), 31-61, doi: $10.1017 /$ S0308210500030742.

[11] M. Fortin, and R. Glowinski, Augmented Lagrangian Methods: Applications to the numerical solution of boundary-value problems, 15, North-Holland, Amsterdam (1983).

[12] D. Henry, Geometric Theory of Semilinear Parabolic Equations, Springer-Verlag, New York (1981), doi: 10.1007/BFb0089647.

[13] J.L. Lions and E. Magenes, Problèmes aux limites non homogènes et applications 1, Dunod, Paris (1968).

[14] A. Matei, Weak solvability via Lagrange multipliers for two frictional contact models, Mathematics and Mechanics of Solids, 21, No. 7 (2014), 826841, doi: $10.1177 / 1081286514541577$.

[15] A. Pazy, Semigroups of Linear Operators and Applications to Partial Differential Equations, Springer-Verlag, New York (1983), doi: 10.1007/978-1-4612-5561-1.

[16] R. Tyrrell Rockafellar, Lagrange multipliers and optimality, SIAM Review, 35, No. 2 (1993), 183-238, doi: 10.1137/1035044.

[17] K. Yosida, Functional analysis, Springer-Verlag, Berlin Heidelberg New York (1980), doi: 10.1007/978-3-642-61859-8.

[18] E. Zeidler, Applied functional analysis : Applications to mathematical physics, SpringerVerlag, New York (1995), doi: 10.1007/978-1-4612-0815-0.

[19] E. Zerrik, A. El Jai and A. Boutoulout, Actuators and regional boundary controllability of parabolic system, Int. J. Syst. Sci., 31, No. 1 (2000), 73-82, doi: 10.1080/002077200291479.

[20] X. Zhang, Exact controllability of semilinear evolution systems and its application, Journal of Optimization Theory and Applications, 107, No. 2 (2000), 415-432, doi: 10.1023/A:1026460831701. 
